\section{Nachhaltige Nutzung der Windenergie}

\author{
Martin Hoppe-Kilpper, Institut für Solare \\ Energieversorgungstechnik (ISET) der Uni- \\ versität Gesamthochschule Kassel
}

Auf dem Gebiet der Windenergienutzung wurde in Deutschland eindrucksvoll unter Beweis gestellt, dass begleitende Programme des Bundes und der Länder, insbesondere aber gesetzliche Maßnahmen wie das Stromeinspeisungsgesetz und in der Nachfolge das Erneuerbare Energien Gesetz, in der Lage sind, technische Entwicklungen und Nutzungsmöglichkeiten einzuleiten, die selbst hochgesteckte Erwartungen weit übertreffen können. Im letzten Jahrzehnt konnten innerhalb von sieben Jahren aufgrund verlässlicher Bedingungen und des technischen Fortschrittes die Anlagengröße fast verzehnfacht, die Anlagenverfügbarkeit auf nahezu $99 \%$ gesteigert und die Stromgestehungskosten etwa halbiert werden. Ende $\mathbf{2 0 0 0}$ waren Windkraftanlagen mit einer Nennleistung von insgesamt $6,1 \mathrm{GW}$ in Deutschland installiert, die mit etwa $2 \%$ zum Nettostromverbrauch beitragen. Auch der Offshore-Nutzung der Windenergie wird in naher Zukunft eine erhebliche Bedeutung zukommen. Derzeit befinden sich mehrere Projekte im GW-Bereich in Planung, die in deutschen Hoheitsgewässern ausgeführt werden sollen.

\section{Einführung}

Forschungs- und Förderprogramme des Bundes sowie der Länder, besonders aber das Stromeinspeisungs- und Erneuerbare Energien Gesetz haben bewirkt, dass Deutschland im internationalen Vergleich mittlerweile einen Spitzenplatz bei der Windenergietechnik und -nutzung einnimmt. So waren im Jahr 2000 in den Ländern der Europäischen Union etwa 13.000 MW Windleistung installiert. Deutschland erreichte mit etwa 6.100 MW davon fast die Hälfte. Zusammen mit der Windleistung weiterer europäischer Länder, in den USA installierten 2.500 MW und den sonstigen, besonders in Asien installierten Anlagen sind weltweit bereits etwa 18.000 MW Leistung errichtet. Die Stromproduktion der Windenergieanlagen in Deutschland im Jahr 2000 betrug bereits $8.600 \mathrm{GWh}$ und übertraf das Vorjahresergebnis um mehr als $40 \%$ (WMEP 1991 - 2001).

Abb. 1: Entwicklung der Windenergienutzung in Deutschland, Europa und weltweit

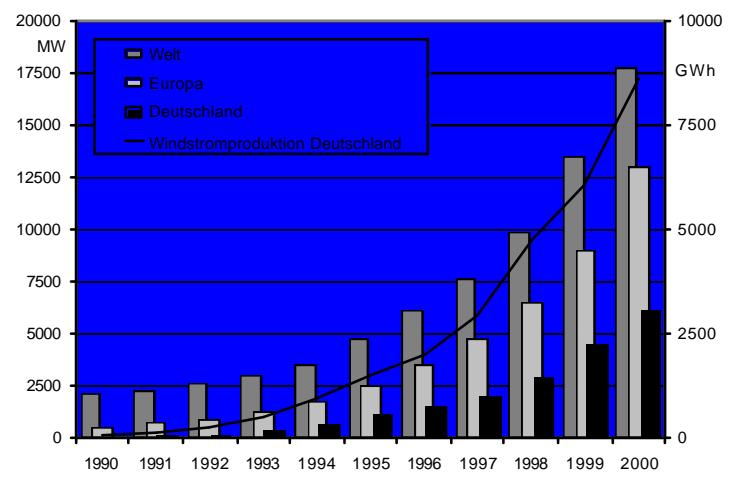

\section{Entwicklung und Stand der Technik}

Ende der 70er Jahre begann die Entwicklung der modernen Windkraftanlagentechnologie in Deutschland und weltweit in zwei sehr unterschiedlichen Leistungsbereichen. Einerseits wurden für private, zumeist landwirtschaftliche Betreiber Anlagen der 10 bis $50 \mathrm{~kW}$-Klasse entwickelt und installiert. Andererseits strebten Energieversorgungsunternehmen eher große Systeme der MW-Klasse an, die jedoch den Prototypstatus nie verließen. Dabei wurden in Deutschland innovative Konzeptionen und Leichtbauweisen bevorzugt. Andere Länder, wie z. B. Dänemark, favorisierten hingegen einfache Systeme in robuster, schwerer Ausführung.

Die Anlagentechnik zu Anfang der 80er Jahre war von vielfältigen Problemen gekennzeichnet, da erprobte Berechnungsmethoden zur Systemauslegung fehlten und nicht auf Erfahrungen aus Konstruktion und Betrieb von Vorläufermodellen zurückgegriffen werden konnte. Weiterhin wurde deutlich, dass die Anlagen mit zunehmender Größe neue technische Herausforderungen mit sich bringen.

$\mathrm{Zu}$ Beginn des ersten Windenergiebooms in Kalifornien standen dem Markt somit zunächst hauptsächlich Anlagen der $50 \mathrm{~kW}$-Klasse zur Verfügung. Ihre Hochskalierung in die 200 $\mathrm{kW}$-Klasse gelang innerhalb weniger Jahre. Bis Ende der 80er Jahre waren dort etwa 16.000 Anlagen mit einer Gesamtleistung von gut 1.500 MW installiert. Allerdings konnten die Verfüg- 
barkeitswerte heutiger Anlagen bei weitem nicht erreicht werden. Die 90er Jahre waren zunächst von der erfolgreichen Einführung der 200 bis $500 \mathrm{~kW}$-Klasse und dem durch das Stromeinspeisegesetz beginnenden Windenergieboom in Deutschland geprägt. Bereits Mitte der 90er Jahre begann die Entwicklung und die Einführung der 1-1,5 MW-Anlagen. Im Frühjahr 2000 wurde die erste 2,5 MW-Turbine neuer Prägung errichtet. Anlagen der 3- bis 5-MWKlasse sind in der Entwic klung.

In allen Leistungsbereichen dominieren mittlerweile die 3-Blatt-Turbinen. Dabei konnten sich - ausgehend von kleinen Einheiten - Anlagen nach dem dänischen Konzept mit Stallregelung, Getriebe und direkt netzgekoppeltem Asynchrongenerator am Markt durchsetzen. Bei größeren Einheiten erreichten in stärkerem Maße Anlagen mit Pitchregelung und drehzahlvariablen Triebstrangkonzepten, insbesondere in getriebeloser Ausführung, wachsende Marktanteile. Somit ist bei MWAnlagen ein deutlicher Trend zu innovativen Konzepten zu erkennen.

Obwohl Windenergieanlagen bereits heute einen hohen technischen Stand aufweisen, gibt es vor allem bei großen Anlagen noch erhebliches Entwicklungspotenzial. Angesichts des verstärkten Ausbaus der Windenergienutzung werden die weitere Steigerung der Zuverlässigkeit, die Erhöhung der Lebensdauer sowie die Verringerung des Wartungsaufwands in $\mathrm{Zu}$ kunft eine größere Rolle spielen. Verbesserte Regelungs- und Überwachungskonzepte bieten dazu einen vielversprechenden Ansatz. Neue Regelungsverfahren können die mechanischen Belastungen von Anlagenkomponenten gezielt reduzieren. Dadurch wird einerseits eine Verlängerung der Lebensdauer erreicht, andererseits kann schon bei der Konstruktion der Anlage die reduzierte Belastung berücksichtigt werden. Besonders bei Anlagen der MWKlasse können dadurch Herstellungskosten reduziert und weitere Verbesserungen der Wirtschaftlichkeit erzielt werden. Weiterhin bieten in Entwicklung befindliche Fehlerfrüherkennungssysteme die Möglichkeit, mechanische Defekte an Windkraftanlagen rechtzeitig zu erkennen, um das Ausmaß von Schäden und die damit verbundenen Belastungen der übrigen Komponenten zu verringern. Darüber hinaus führt der Einsatz solcher Verfahren zu einer Reduktion des Wartungsaufwands und der Stillstandszeiten. Somit lassen sich auch wesentliche Voraussetzungen für einen OffshoreEinsatz großer Turbinen schaffen.

\section{Wirtschaftlichkeit und Stromgestehungs- kosten}

Der weitaus größte Teil der in Deutschland errichteten Windenergieanlagen wird mit besonderen, zinsgünstigen Darlehen für Umweltschutzmaßnahmen finanziert. Das Bundesministerium für Wirtschaft gewährt zur Finanzierung umweltrelevanter Maßnahmen, z. B. Windenergieanlagen, über die Deutsche Ausgleichsbank langfristige, zinsvergünstigte Darlehen, die etwa $1 \%$ unter dem üblichen Marktzins liegen. Die Auszahlungshöhe ist abhängig vom prozentualen Darlehensanteil an der Cesamtinvestition und kann bis zu $100 \%$ betragen. Die ersten beiden Jahre sind tilgungsfrei, der Zins ist für die gesamte Dauer des Darlehens, im allgemeinen zehn Jahre, fest. Für das in Abbildung 2 dargestellte Berechnungsbeispiel wurde mit einem resultierenden Zinssatz von $5,5 \%$ gerechnet, der sich aus einem $25 \%$-igen Eigenkapitalanteil zu $7 \%$ und einem entsprechenden Fremdkapitalanteil zu $5 \%$ zusammensetzt. 
Abb. 2: Stromgestehungskosten für Windenergieanlagen

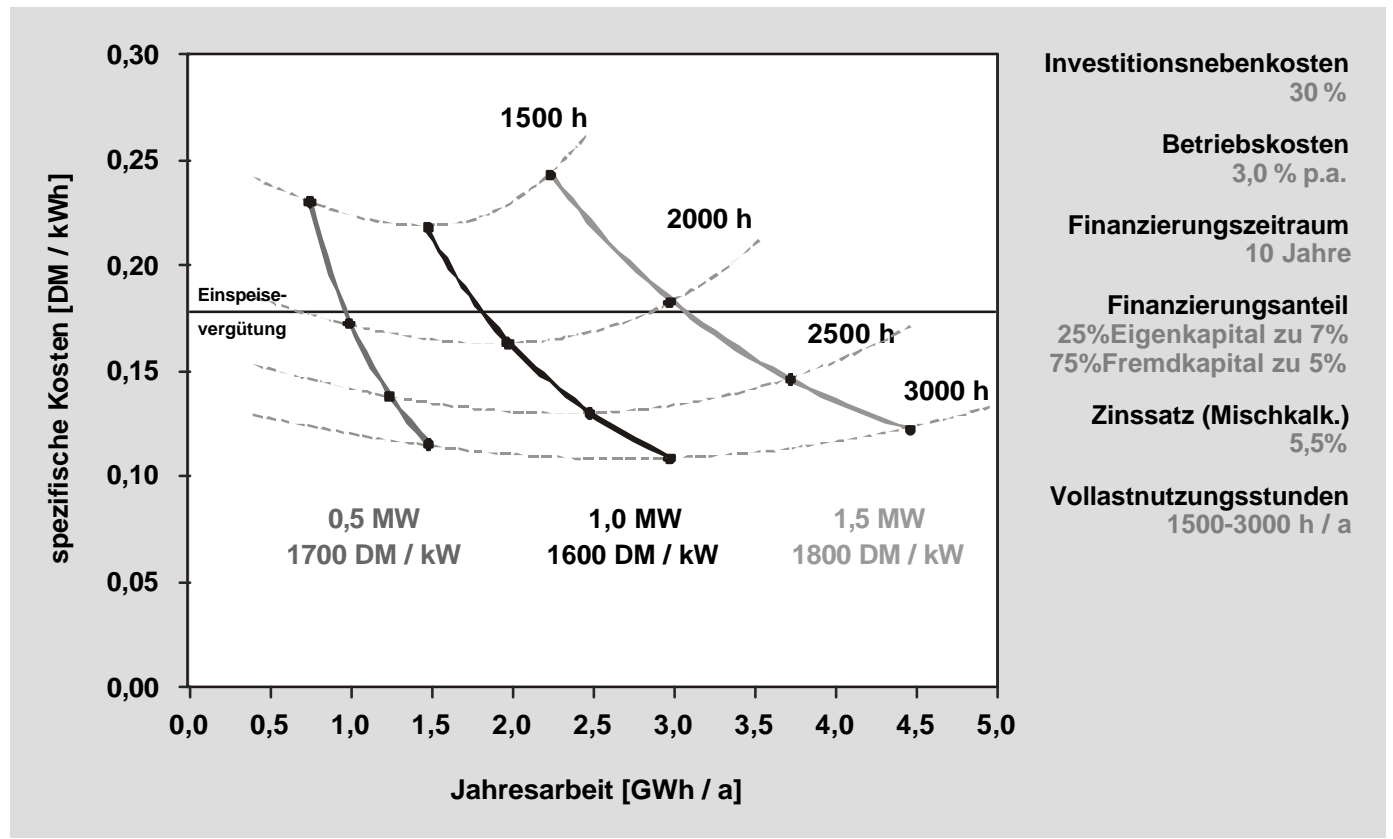

Unter Berücksichtigung der in Abbildung 2 genannten Kostenarten ergeben sich Stromgestehungskosten in DM pro Kilowattstunde für die Stromerzeugung aus Windenergie, die maßgeblich von den spezifischen Kosten der eingesetzten Anlagentypen abhängen. In Bild 2 sind exemplarisch für Anlagen der 0,5 MWKlasse sowie für 1,0 MW- und 1,5 MWAnlagen jeweils die berechneten Stromgestehungskosten über der Bezugsgröße ,Jahresarbeit" dargestellt. Der Wertebereich der Kurvenscharen umfasst Volllastnutzungsstunden von jeweils 1.500 bis $3.000 \mathrm{~h} / \mathrm{a}$ und charakterisiert somit eher windgünstige Standorte. Nach den bisherigen Erfahrungen mit Windenergieanlagen bis etwa $1.000 \mathrm{~kW}$ Nennleistung werden bei zunehmender Anlagengröße günstigere Stromgestehungskosten erzielt. Diese Tendenz wird jedoch bei den Anlagentypen oberhalb von 1 MW noch nicht fortgeschrieben. Die spezifischen Stromgestehungskosten für diese Anlagenklasse liegen zur Zeit etwa $10 \%$ uiber denen der 0,5- bzw. 1,0-MW-Klasse (Durstewitz et al. 1998).

\section{Windenergie in einer dezentralen Energie- versorgung}

Dezentrale Energieversorgung umfasst sowohl den netzparallelen als auch den autonomen Betrieb von kleinen, modularen Erzeugungsanlagen durch Energieversorgungsunternehmen, deren Kunden, oder aber davon unabhängigen Dritten (Kleinkauf et al. 1997). Das dezentrale Konzept unterscheidet sich dabei fundamental vom zentralen Versorgungsansatz. Die Erzeugungseinheiten können nämlich auch direkt am Verbrauchspunkt betrieben werden und damit entweder zur unmittelbaren Bedarfsdeckung eines zugeordneten Verbrauchers oder aber zum Nutzen des gesamten dezentralen Versorgungssystems beitragen.

Zukünftige Versorgungssysteme werden zunehmend daran gemessen werden, in wie weit ein effizienter, ökonomischer Betrieb und die Forderungen zur Nachhaltigkeit miteinander in Einklang gebracht werden. Im Detail sollten zukünftige Versorgungssysteme folgende Anforderungen erfüllen:

- Zuverlässiger und kostengünstiger Betrieb

- Hohe Effizienz hinsichtlich Umweltverträglichkeit und Ressourcenverbrauch

- Möglichkeit zur regionalen Nutzung erneuerbarer Energien 
- Modulare Erweiterbarkeit

- Kurze Planungs-, Genehmigungs- und Errichtungszeiten.

Dezentrale, verbrauchernahe Energieversorgung bedeutet nicht, dass zukünftig nur noch separierte (autonome) Verteilungsnetze auf Mittel- oder Niederspannungsebene die Stromversorgung einzelner Regionen oder Städte sicherstellen sollen und keine Übertragungsnetze auf Höchstspannungsebene mehr benötigt würden (Ensslin u. Hoppe-Kilpper 2000). Obwohl es derartige Versorgungslösungen durchaus geben kann, wird das Übertragungsnetz für einen Ausgleich zwischen entfernteren Regionen sowie für Stromimporte aus dem Ausland weiterhin seine Bedeutung behalten. Nur durch eine elektrische Verbindung - auch über längere Distanzen - können die regionalen Ressourcen an erneuerbaren Energien umfassend genutzt werden. So müssen schon heute zeitweilig Windleistungen aus den deutschen Küste ngebieten mit Hilfe des Übertragungsnetzes in andere Regionen transportiert werden. Dabei wird in Schwachlastzeiten bei gleichzeitig hohen Windgeschwindigkeiten aus dem schleswig-holsteinischen Mittelspannungsnetz ins vorgeschaltete Übertragsnetz zurïckgespeist. Möchte man auf derartige Möglichkeiten verzichten, müssten zwangsläufig die Windenergieanlagen abgeregelt werden, mit entsprechend negativen Auswirkungen auf die Auslastung. Dieser Aspekt wird sich noch drastisch verstärken, wenn die immensen OffshorePotenziale in Nord- und Ostsee erschlossen werden. So sieht ein Szenario des Bundesministeriums für Umwelt und Reaktorsicherheit (BMU) vor, bis zum Jahr 2030 mit ca. $25 \mathrm{GW}$ installierter Windleistung rund $16 \%$ des deutschen Stromverbrauchs allein aus OffshoreWindenergie zu decken. Diese Leistungen können nur dann effektiv genutzt werden, wenn sie auch in die Verbrauchszentren, z. B. an Rhein und Ruhr, transportiert werden.

Hinsichtlich des Nutzungsgrads erneuerbarer Energien lassen sich bei dezentralen Versorgungskonzepten drei unterschiedliche Kategorien definieren:

- Regionen mit einem Überangebot an erneuerbaren Energien und entsprechendem Potenzial zur Energielieferung in andere Regionen,
- Regionen mit einem Unterangebot an $\mathrm{er}$ neuerbaren Energien und entsprechender Notwendigkeit zum Energiebezug aus anderen Regionen,

- Regionen mit einer nahezu ausgeglichenen Bilanz erneuerbarer Energien.

\section{Nutzung von großen, lokalen Windenergie- Potenzialen}

Neben der dezentralen, verbrauchernahen Nutzung der Windenergie, sollten darüber hinaus auch die ausgesprochen großen Potenziale an den Standorten genutzt werden, an denen keine unmittelbaren Verbraucher vorhanden sind. Dies gilt sowohl für die Nutzung der WindPotenziale im europäischen wie im außereuropäischen Raum (z. B. im Sonnengürtel Afrikas) als auch für die Offshore-Windenergie in der deutschen Ausschließlichen Wirtschaftszone (AWZ) ${ }^{1}$.

\section{Nutzung großer Offshore-Potenziale}

Sollten z. B. im Rahmen der nationalen $\mathrm{CO}_{2}$ Senkungsstrategien die gewaltigen OffshoreWindenergie-Potenziale in der AWZ tatsächlich realisiert werden, so ist ein Transport über große Entfernungen (auch an Land) und demnach eine elektrische Anbindung ans Höchstspannungsnetz naheliegend. Diese Einspeisungen im Gigawatt-Bereich würden mittel- bis langfristig die dort noch bestehenden thermischen Kraftwerkskapazitäten ersetzen. Als dargebotsabhängige Energiequelle ergeben sich dann jedoch besondere Anforderungen an die Betriebsweise dieser großen OffshoreWindparks. Besonders zu nennen wären dabei unter anderem:

- Regelbarkeit der abgegebenen Wirk- und Blindleistung (auch durch den zuständigen Netzbetreiber)

- Freischaltmöglichkeit für Wartungsarbeiten an Windenergieanlagen und Netz (auch durch den zuständigen Netzbetreiber)

- Einsatz leistungsfähiger Prognose-Programme für zu erwartende Energielieferungen

- $\quad$ Optimierte Fernüberwachung und -wartung 
- $\quad$ Sicherstellung ausreichender Arbeitsbedingungen für Betriebs- und Wartungspersonal (Aufenthalts- und Übernachtungsräume)

- Klärung rechtlicher Probleme im Zusammenhang mit Eigentumsfragen und notwendigem externen Zugriff auf Anlagenbetrieb durch Netzbetreiber.

\section{Nutzung großer Wind-Potenziale im inter- kontinentalen Netzverbund}

Das Windenergieangebot ist bekanntlich groBen saisonalen Schwankungen unterworfen. So befindet sich Europa klimatisch betrachtet in einer typischen Winterwindregion. Hier liegen z. B. die langfristigen Julimittelwerte der potenziellen Windstromerzeugung bei unter $40 \%$ der potenziellen Windstromerzeugung im Januar. Im Hinblick auf eine saisonal ausgeglichene Stromversorgung mit Windenergie kommen als Ergänzung besonders die Passatwindregionen Nordafrikas in Frage, da zwischen Passatwindregionen und Winterwindregionen eine deutliche Antikorrelation besteht. Ein weitgehender jahreszeitlicher Ausgleich kann somit schon durch die großräumige Nutzung von Standorten in der selben Hemisphäre erreicht werden.

Es stellt sich somit die Frage, welche Vorteile eine sehr großräumige (interkontinentale) Stromversorgung haben könnte. In einer Studie (Czisch et al. 1999) wurde die gleichzeitige Nutzung von 20 ausgewählten marokkanischen und algerischen Onshore-Gebieten sowie von 47 europäischen Offshore-Gebieten untersucht. Dabei wird angenommen, dass sich $1 / 3$ der installierten Leistung in Nordwestafrika und $2 / 3$ Offshore in Europa befinden. Die größte Leistungsvergleichmäßigung lässt sich erwartungsgemäß bei gemeinsamer Nutzung aller 67 Gebiete erzielen. Die Summenleistung unterschreitet dabei $20 \%$ der Nennleistung nur noch in $2 \%$ eines Jahres und die niedrigste Leistung, die erreicht wird, liegt bei $12 \%$. Werte von über $70 \%$ sind jedoch fast vernachlässigbar. Eine gegenüber regionaler Nutzung erhöhte Minimalleistung hat zur Folge, dass der Windenergie ein größerer Teil der installierten Leistung als annähernd gesichert angerechnet werden kann. Durch den damit steigenden Kapazitätseffekt reduziert sich auch die nötige Reserveleistung aus anderen Kraft- werken. Durch eine ergänzende, großräumige Nutzung der Windenergie lassen sich demnach die dargebotsabhängigen Eigenschaften einer regionalen Erzeugung deutlich vermindern.

\section{Zusammenfassung und Ausblick}

Die großtechnische Anwendung der Windenergie beschränkt sich im Allgemeinen auf die Elektrizitätserzeugung. In Deutschland wurde eindrucksvoll unter Beweis gestellt, dass begleitende Programme des Bundes und der Länder, insbesondere aber gesetzliche Maßnahmen wie das Stromeinspeisungsgesetz, in der Lage sind, technische Entwicklungen und Nutzungsmöglichkeiten einzuleiten, die selbst hochgesteckte Erwartungen weit übertreffen können. Im letzten Jahrzehnt konnten innerhalb von sieben Jahren aufgrund verlässlicher Bedingungen und des technischen Fortschritts die Anlagengröße fast verzehnfacht, die Anlagenverfügbarkeit auf nahezu $99 \%$ gesteigert und die Stromgestehungskosten etwa halbiert werden. Ende 2000 waren Windkraftanlagen mit einer Nennleistung von insgesamt $6,1 \mathrm{GW}$ in Deutschland installiert, die mit etwa $2 \%$ zum Nettostromverbrauch beitragen. Damit ist die Windenergietechnik - zumindest regional - in Deutschland $\mathrm{zu}$ einem Wirtschaftsfaktor geworden und hat insgesamt mehr als 30.000 Arbeitsplätze geschaffen. Diesen Erfolg auszubauen und zu sichern wird nur gelingen, wenn sich - auf den heimischen Absatz gestützt auch ein weltweiter Exportmarkt erfolgreich entwickeln kann.

Auch der Offshore-Nutzung der Windenergie wird in naher Zukunft eine erhebliche Bedeutung zukommen. Derzeit befinden sich mehrere Projekte im GW-Bereich in Planung, die in deutschen Hoheitsgewässern ausgeführt werden sollen.

Die weitere Nutzung der Windenergie in relevanten Größenordnungen bei weiträumig verteilten Anlagen wird auch davon abhängen, wie exakt die Einspeiseleistung in das Verbundnetz vorausgesagt und welche Beiträge zur Netzstützung geliefert werden können. Durch genaue Vorhersage der Windleistung und durch Gestaltung der Einspeiseleistung entsprechend der Netzanforderungen (z. B. Ausgleich durch weiträumige Verteilung oder Einbindung von 
Speichern) kann der Marktwert der Windenergie entscheidend erhöht werden (Rohrig 2000).

\section{Anmerkung}

1) Die Zone seewärts der 12-Seemeilen-Zone, welche das Küstenmeer begrenzt.

\section{Literatur}

Czisch, G.; Durstewitz, M.; Hoppe-Kilpper, M.; Kleinkauf, W., 1999: Windenergie gestern, heute und morgen. Husum Wind, Husum

Durstewitz, M.; Hoppe-Kilpper, M.; Kleinkauf, W., 1998: Allgemeine Entwicklung der Kosten der Windstromerzeugung in Deutschland. Studie im Auftrag des Bundesverband Windenergie e.V., ISET Kassel

Ensslin, C.; Hoppe-Kilpper, M., 2000: Elektrische Energieversorgung mit hohem Anteil dezentraler und regenerativer Stromerzeugung. Kurzstudie im Auftrag des Deutschen Bundestags - Ausschuss für Bildung, Forschung und Technikfolgenabschätzung, ISET Kassel

Kleinkauf, W.; Raptis, F.; Haas, O., 1997: Elektrifizierung mit erneuerbaren Energien. In: Themenheft 96/97, Forschungsverbund Sonnenenergie (FVS), Köln

Rohrig, K., 2000: Online-Monitoring and ShortTerm Prediction of 2400 MW Wind Power in an Utility Supply Area. IEA Expert Meeting "Wind Forecasting Techniques“. Boulder, USA

WMEP 1991 - 2001: Wissenschaftliches Mess- und Evaluierungsprogramm zum Breitentest ,250 MW Wind“ - Jahresauswertungen 1990 bis 2000. ISETSchriftenreihe, Kassel

\section{Kontakt}

Dipl.-Ing. Martin Hoppe-Kilpper

Leiter des Forschungsbereichs Information und

Energiewirtschaft

Institut für Solare Energieversorgungstechnik e.V. (ISET)

Königstor 59, 34119 Kassel

Tel.: +49 (0) $561 / 7294$ - 341

Fax: +49 (0) 561 / 7294 - 260

E-Mail: m.hoppe-kilpper@iset.uni-kassel.de

URL: $\quad$ www.iset.uni-kassel.de

\section{Nachhaltige Nutzung von Bio- masse als Energieträger}

\author{
Christine Rösch, ITAS
}

Die EU-Mitgliedsstaaten haben sich verpflichtet, während der nächsten zehn Jahre Ziele für den künftigen Inlandsverbrauch von Energie aus regenerativen Energieträgern festzulegen und jährlich zu erfüllen. Maßstab für die Zielsetzung sind das Weißbuch der EU-Kommission ${ }^{1}$ und die Kyotoverpflichtungen (EU-Kommission 2000a). Die nachfolgenden Ausführungen zeigen, dass die Biomasse über große technische Entwicklungspotenziale verfügt und wesentliche Beiträge zur Erreichung dieses ambitionierten Zieles leisten kann, ohne dabei andere Nachhaltigkeitsanforderungen zu verletzen. Einer raschen weitergehenden Erschließung dieses Potenzials stehen im Allgemeinen weniger technische und ökologische als vielmehr ökonomische Hemmnisse entgegen.

\section{Stand der Biomassenutzung in der EU und in Deutschland}

Der Beitrag der Biomasse ${ }^{2}$ zur Deckung des EU-Energieverbrauchs umfasste 1998 etwa $3 \%$ (EU-Kommission 2000b). Deutlich über diesem EU-Durchschnitt liegt ihr Anteil in Österreich (12\%), Schweden (18\%) und in Finnland (23\%). Beim überwiegenden Teil der energetisch genutzten Biomasse handelt es sich um Schwachholz und Waldrestholz aus der Forstwirtschaft, Industrierestholz aus der Holzwirtschaft und Altholz.

Die Biomasse leistet damit gegenwärtig den größten Beitrag zur Bereitstellung erneuerbarer Energie in der EU. Sie stellt rd. $64 \%$ der Primärenergie aus regenerativen Energien (das entspricht 54,18 Mio. t Rohöleinheiten - RÖE ${ }^{3}$ ). Und ihre Bedeutung nimmt weiter zu: Im Zeitraum 1995 bis 1998 ist die Bereitstellung von Primärenergie durch Biomasse in der EU um $13,5 \%$ gewachsen; in einzelnen Mitgliedsstaaten lag dieser Zuwachs deutlich darüber (z. B. $75 \%$ in Deutschland und $94 \%$ in Italien).

In Deutschland trägt die Biomasse derzeit nur zu etwa rd. $1 \%$ - bezogen auf den fossilen Primärenergieeinsatz in Höhe von 14,3 EJ im Jahr 1998 - zur Deckung der Energienachfrage 